\title{
Attentional Modulation of the Inner Ear: A Combined Otoacoustic Emission and EEG Study
}

\author{
Anna Wittekindt, Jochen Kaiser, and Cornelius Abel \\ Institute of Medical Psychology, Goethe University Frankfurt, 60528 Frankfurt am Main, Germany
}

\begin{abstract}
Attending to a single stimulus in a complex multisensory environment requires the ability to select relevant information while ignoring distracting input. The underlying mechanism and involved neuronal levels of this attentional gain control are still a matter of debate. Here, we investigated the influence of intermodal attention on different levels of auditory processing in humans. It is known that the activity of the cochlear amplifier can be modulated by efferent neurons of the medial olivocochlear complex. We used distortion product otoacoustic emission (DPOAE) measurements to monitor cochlear activity during an intermodal cueing paradigm. Simultaneously, central auditory processing was assessed by electroencephalography (EEG) with a steady-state paradigm targeting early cortical responses and analysis of alpha oscillations reflecting higher cognitive control of attentional modulation. We found effects of selective attention at all measured levels of the auditory processing: DPOAE levels differed significantly between periods of visual and auditory attention, showing a reduction during visual attention, but no change during auditory attention. Primary auditory cortex activity, as measured by the auditory steady-state response (ASSR), differed between conditions, with higher ASSRs during auditory than visual attention. Furthermore, the analysis of cortical oscillatory activity revealed increased alpha power over occipitoparietal and frontal regions during auditory compared with visual attention, putatively reflecting suppression of visual processing. In conclusion, this study showed both enhanced processing of attended acoustic stimuli in early sensory cortex and reduced processing of distracting input, both at higher cortical levels and at the most peripheral level of the hearing system, the cochlea.
\end{abstract}

Key words: alpha oscillation; auditory steady-state responses; cochlea; DPOAE; selective attention

\section{Introduction}

Everyday life in complex multisensory environments requires the ability to selectively attend to a specific subset of incoming pieces of sensory information while ignoring others. This can be achieved by selectively directing attentional resources to single modalities or to distinct features of a stimulus. The attentional gain control leads to an enhanced processing of the selected stimulus reflected by faster reaction times and higher response accuracies (Ward, 1997; Carrasco et al., 2004).

The mechanism underlying attentional gain control might consist of a neuronal enhancement of relevant information or a suppression of distractors (Fritz et al., 2007; Couperus and Mangun, 2010). It is still a matter of debate regarding at which level of the processing hierarchy in the CNS selective attention exerts its influence. Electrophysiological studies in humans have concluded that attention affects the initial stages of cortical processing (Karns and Knight, 2009; Saupe et al., 2009), but not

\footnotetext{
Received Nov. 19, 2013; revised May 18, 2014; accepted June 9, 2014.

Author contributions: A.W., J.K., and C.A. designed research; A.W. and C.A. performed research; A.W. and C.A. analyzed data; A.W., J.K., and C.A. wrote the paper.

This work was supported by an intramural young investigator grant from the medical faculty, Goethe University Frankfurt.

The authors declare no competing financial interests.

Correspondence should be addressed to Cornelius Abel, Ph.D., Institute of Medical Psychology (IMP), Johann Wolfgang Goethe University, Heinrich-Hoffmann-Str. 10, 60528 Frankfurt am Main, Germany. E-mail: C.Abel@med.uni-frankfurt.de.

DOI:10.1523/JNEUROSCI.4861-13.2014

Copyright $\odot 2014$ the authors $\quad 0270-6474 / 14 / 349995-08 \$ 15.00 / 0$
}

subcortical stages (Picton et al., 1971; Connolly et al., 1989). In contrast, previous researchers highlighted that the most peripheral stage of the hearing system, the cochlea, could be modulated by attention (Meric and Collet, 1994; Giard et al., 2000; de Boer and Thornton, 2007). Several human and animal studies have shown that sound processing in the peripheral auditory system, investigated by otoacoustic emissions (Puel et al., 1988; Maison et al., 2001; Smith et al., 2012), cochlear microphonics (Delano et al., 2007), or auditory brainstem potentials (Lukas, 1980), is modulated during tasks requiring focused attention on either auditory or visual stimuli. However, the described effects were small and sometimes contradictory (Avan and Bonfils, 1992; but see negative results by Michie et al., 1996).

Physiologically, the peripheral effects of attention are supposed to be mediated by the medial olivocochlear (MOC) efferent system, consisting of neurons that originate in the brainstem, project to the cochlea, and innervate the outer hair cells (Guinan, 1996; Russell and Lukashkin, 2007). Outer hair cells are the core element of the cochlear amplifier because they amplify the basilar membrane vibrations of low-level sounds by actively changing their cell length and thereby reinforcing the vibration (Robles and Ruggero, 2001; Ashmore, 2008). Therefore, the olivocochlear efferent system can modulate the sound amplification characteristics of the cochlea.

The aim of the present study was to investigate intermodal selective attention in humans at different levels of the auditory system simultaneously. To access peripheral effects, we used dis- 

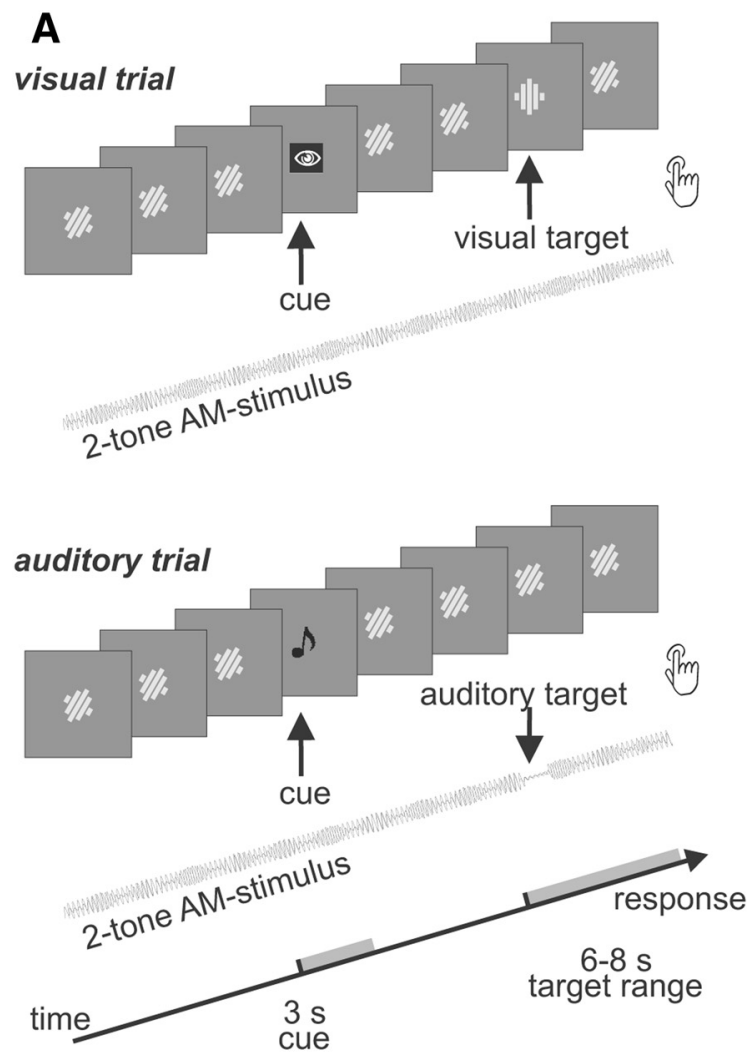

B
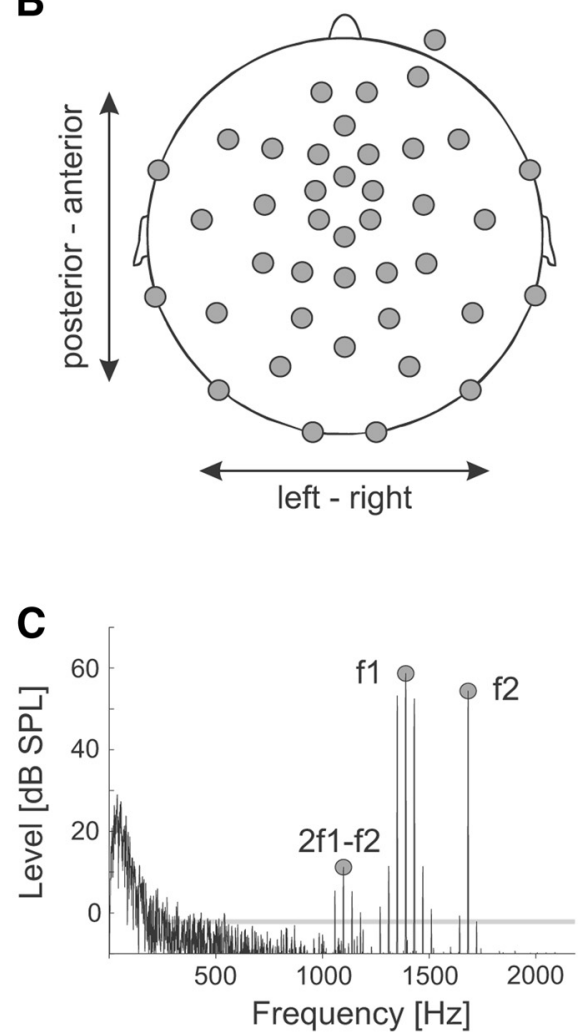

Figure 1. Schematic illustration of the task and the EEG layout. $A$, Paradigm: Simultaneous acoustic ( $40 \mathrm{~Hz}$ amplitude modulated two-tone stimulus) and visual (Gabor patch) stimulation. After $3 \mathrm{~s}$, a visual cue indicated the to-be-attended modality (visual or auditory). The target (stimulus deviation) occurred in the time range of $3-5 \mathrm{~s}$ after the cue and had to be responded to by an immediate button press. $\boldsymbol{B}$, EEG electrode layout with 41 electrodes. $C$, Exemplary frequency spectrum of the DPOAE measurement including the primary tones ( $\mathrm{f} 1: 1390 \mathrm{~Hz}$; $\mathrm{f} 2: 1682 \mathrm{~Hz})$ and the 2f1-f2 DPOAE. The noise level is marked by the gray line.

tortion product otoacoustic emissions (DPOAE) to reveal the cochlear activation state (Kemp, 2007). Simultaneously, we measured neuronal activity by electroencephalography (EEG) and analyzed the auditory steady-state response (ASSR) as a marker of processing in the primary auditory cortex (Gutschalk et al., 1999; Engelien et al., 2000). Furthermore, we investigated oscillatory neuronal activity (alpha-band activity), which might reflect the impact of attentional processes at higher cortical levels (Foxe and Snyder, 2011; Klimesch, 2012). To our knowledge, our study is the first that simultaneously tested changes of DPOAEs and cortical activity in the context of selective attention.

\section{Materials and Methods}

Participants. Twenty-three healthy volunteers (13 females, age range: 20-39 years) participated in this study. All participants were righthanded and did not report any known hearing deficit. Subjects received a remuneration of 10 Euros per hour. All subjects were informed about the experimental procedure and the aims of the study and gave written informed consent. The study was approved by the Ethics Committee of the University of Frankfurt Medical Faculty.

Experimental paradigm. We investigated intermodal selective attention and measured cochlear and neuronal responses simultaneously by recording DPOAE and EEG, respectively. To ensure highly controlled attentional conditions, we did not use a block design as in previous otoacoustic emission studies in humans (Puel et al., 1988; Froehlich et al., 1993; Smith et al., 2012), but rather chose a paradigm in which a cue predicted the modality (auditory or visual) to attend in each trial, a common approach for behavioral investigations of attention (Ward, 1997; Green and McKeown, 2001; Carrasco et al., 2004).

Measurements were performed in a sound-attenuated, electrically shielded chamber (Industrial Acoustic Company) in which subjects sat quietly on a comfortable chair. The visual stimulus was a continuously displayed Gabor patch (diameter: 3.3 degrees of visual angle; 2.1 cycles per degrees) of low contrast presented on a computer screen placed outside of the chamber behind an electrically shielded window (distance: $1.4 \mathrm{~m}$ ). The acoustic stimulus was a continuous stimulus consisting of 2 simultaneously presented tones (duration 6-8 s, half cosine ramp: 25 ms, for details, see Acoustic Measurement and Analysis section below) presented binaurally via ear inserts.

The paradigm was designed in the style of classical cueing paradigms (Posner, 1980) with two experimental conditions, Attend Auditory and Attend Visual, in a pseudorandomized order. Figure $1 A$ illustrates the experimental paradigm schematically. Each trial started with a period of nondirected attention. After $3 \mathrm{~s}$, a cue was shown ( $0.5 \mathrm{~s}$ duration) on the computer screen, indicating the to-be-attended modality. An eyesymbol indicated the Attend Visual condition and a musical note indicated the Attend Auditory condition. During the following time period, the participants had to attend selectively to the indicated modality. The task was to detect a target in the selected modality appearing 3-5 s after the cue. The cue was $100 \%$ informative (valid) to avoid divided attention and to achieve a maximal focusing of attention. The target in the visual modality was a slight turn (angle change) of the Gabor patch; the target in the auditory modality was a short $(200 \mathrm{~ms})$ level decrease of the amplitude-modulated stimulus at the DPOAE recording ear. The participant was requested to react immediately by pressing a button with the right index finger when detecting the target. The time window to respond lasted for $0.85 \mathrm{~s}$ after target appearance. Slower responses were counted as misses. Consecutive trials were separated by a fixed intertrial time interval of $1.7 \mathrm{~s}$ with no auditory or visual stimulation.

Five blocks of 50 trials were performed. In each of the blocks, the difficulty of the task was adjusted individually by a staircase method to ensure high challenge and comparable demands between conditions and between subjects. The target deviation ranged between a level change of 
$1-12 \mathrm{~dB}$ for the auditory task and an angle change of $0.5-12^{\circ}$ for the visual task.

Acoustic measurement and analysis. DPOAEs were measured in one of the two ears (right or left ear balanced between the subjects) using a probe assembly, which incorporates a sensitive microphone and two loudspeakers (ER-10C microphone/preamplifier system; Etymotic Research) placed into the subject's meatus with a foam ear tip. The microphone signal was preamplified $(+40 \mathrm{~dB})$ and fed into an A/D-recording interface (Edirol FA-66; Roland). The input and output channels of the sound card were sampled at a rate of $48 \mathrm{kHz}$. To present the defined stimulus levels, the two loudspeakers of the probe assembly were separately calibrated in the test ear immediately before the measurements started by measuring the frequency response of a white noise stimulus. Acoustic stimuli on the non-DPOAE ear were presented via an airconducting tube with an ear-inserted foam tip (E-A-RTONE 3A; 3M). To ensure equal sound pressure levels (SPLs), all loudspeakers were calibrated using a sound level meter (120-0014; REED Instruments). Digital stimulus generation and data acquisition were controlled by custom-written MATLAB scripts (version 7.10; The MathWorks).

DPOAEs are generated when stimulating the ear with two pure tones of different frequencies ( $\mathrm{f} 1$ and $\mathrm{f} 2, \mathrm{f} 1<\mathrm{f} 2$ ) and appropriate $\mathrm{f} 2 / \mathrm{f} 1$ frequency ratios delivered through two separate loudspeakers. The $\mathrm{f} 2 \mathrm{fre}-$ quency was set between 1000 and $2000 \mathrm{~Hz}$ and was adjusted in each participant to the frequency evoking the highest DPOAE level. The frequency ratio $\mathrm{f} 2 / \mathrm{f} 1$ was 1.21 and the levels of the two primary tones were $65 \mathrm{~dB}$ SPL (f1) and $55 \mathrm{~dB}$ SPL (f2). To evoke auditory steady-state responses, the f1 tone was additionally amplitude-modulated with a modulation frequency of $40 \mathrm{~Hz}$ and $100 \%$ modulation depth.

DPOAE levels were determined for each trial separately by calculating the frequency spectrum using a fast Fourier transform (FFT, Hanning window) and taking the amplitude at the 2f1-f2 DPOAE frequency. To obtain the time course of DPOAEs across trials, we used a moving window method (window size: $1000 \mathrm{~ms}$, shifted from $0-6 \mathrm{~s}$ in steps of 250 $\mathrm{ms}$ ). The background noise level in the test ear was calculated as the mean sound level at frequencies around the DPOAE frequency (10 data points below and above $2 \mathrm{f} 1-\mathrm{f} 2$ ). Only clearly measurable DPOAEs, defined as 2f1-f2 levels above mean noise level plus two SDs, were taken into account for further analysis. Single time points with atypical high background noise, for example, those caused by swallowing, were excluded from the analysis (mean rate of rejected data points: $15.26 \%$, median: $10.08 \%)$.

For each participant, the time course of DPOAEs was averaged across trials for both conditions (Attend Visual and Attend Auditory). However, because it is known that DPOAEs show variations over time (Wagner et al., 2008) and the focus of our study was on small DPOAE level changes following the informative cue within each trial, the time period before cue (2-3 s) was taken as a baseline and DPOAE levels were expressed relative to the mean baseline level before averaging across trials.

To analyze the difference between the two selective attention conditions statistically, the DPOAE level was additionally calculated for two longer time windows: before selective attention (defined as the period from 1-3 s after stimulus onset) and during selective attention (defined as the period from 3.5-5.5 s after stimulus onset). The effect of intermodal attention on the otoacoustic emissions, defined as the difference between the DPOAE level during selective attention minus the DPOAE level before selective attention was compared statistically between the two conditions using standard $t$ tests. When calculating this difference between the time periods during and before selective attention, only trials that did not show atypical high background noise in the period before or during selective attention (mean rate of rejected trials per subject: $18.83 \%$, median: $16 \%$ ) were used.

Electrophysiological recording and analysis. EEG was recorded continuously with a 128-channel QuickAmp amplifier (Brain Products) and $124 \mathrm{Ag}-\mathrm{AgCl}$ electrodes mounted in an elastic cap (Falk Minow Services). In the present study, 41 electrodes [including two electrooculography (EOG) channels] were used (Fig. 1B), covering approximately the whole head in concentric shapes with additional electrodes in the frontocentral region, where auditory steady-state responses are expected (Griskova et al., 2009; Saupe et al., 2009b). Two electrodes were mounted infraorbit- ally and supraorbitally to the right eye to monitor eye blinks and eye movements. The electrode array was digitized for each participant after the recording using an electrode-localization system (Zebris/ElGuide). All channels were recorded with an average reference, a forehead ground, and impedances of $<\sim 10 \mathrm{k} \Omega$. The EEG was digitized with a sampling rate of $1000 \mathrm{~Hz}$ and an anti-aliasing filter of $135 \mathrm{~Hz}$.

The preprocessing of the EEG data and the statistical analyses were performed with MATLAB (Version 8.0) and the open-source FieldTrip toolbox for EEG/MEG analysis (Oostenveld et al., 2011). The signal was band-pass filtered $(0.1-150 \mathrm{~Hz})$ and re-referenced to the average of the two mastoid electrodes. Epochs of $7.5 \mathrm{~s}$, starting $1000 \mathrm{~ms}$ before stimulus onset, were extracted. Trials with artifacts were rejected by an automatic approach: trials with muscle artifacts were detected by means of thresholding the $z$-transformed value of the preprocessed raw data (110-150 $\mathrm{Hz}$ band-pass filter, Hilbert analytic amplitude, $z$-score cutoff $=20)$ and rejected. Additional artifacts were defined as voltage jumps exceeding 60 $\mu \mathrm{V}$. First channels with $>20$ artifact-contaminated trials were fully excluded. After that, remaining trials with artifacts were rejected. The mean rate of remaining trials after artifact rejection across all participants was $87.9 \%$ (visual trials: $88.3 \%$; auditory trials: $87.6 \%$ ). To remove eye artifacts, the data underwent an independent component analysis (runica) and components correlating with the EOG channels $(r>0.3)$ were automatically rejected.

For ASSR estimation, epochs were averaged per participant, condition, and electrode site thus emphasizing stimulus-locked activation while suppressing induced brain responses and non-brain-related noise. The resulting event-related field was transformed into a time-frequency response using Hanning tapers with a fixed time window of $1000 \mathrm{~ms}$, shifted from -1 to $6 \mathrm{~s}$ poststimulus onset in steps of $50 \mathrm{~ms}$, and power was calculated by FFT. Because the relevant frequency was defined by the modulation frequency of the evoking stimulus, temporal power profiles and topographic distribution were calculated only for the $40 \mathrm{~Hz}$ (ASSR) component.

In addition to the evoked auditory steady-state response, stimulusinduced oscillatory activity was estimated by first analyzing the signal by time frequency on a single-trial basis (Hanning taper, time window: 500 ms; moving window from -1 prestimulus to $6 \mathrm{~s}$ poststimulus onset in steps of $50 \mathrm{~ms}$ ) and then averaging across the trials. Alpha activity was defined as the mean power of the frequency band between 8 and $14 \mathrm{~Hz}$. Statistical analysis of alpha and ASSR power was performed by nonparametric cluster-based permutation tests (Maris and Oostenveld, 2007).

\section{Results}

Performance was very similar for both conditions. The average hit rates were $82.99 \%$ ( $\pm 0.04 \mathrm{SD}$ ) for the visual task and $82.47 \%$ $( \pm 0.05 \mathrm{SD})$ for the auditory task. They did not differ between conditions (paired $t$ test: $t_{(22)}=0.71, p=0.48$ ). False alarm rates (visual: $1.29 \%$, auditory: $1.32 \%$ ) and missed trials (visual: $15.72 \%$, auditory: $16.21 \%$ ) were also comparable between conditions and did not show significant differences (paired $t$ tests for false alarms: $t_{(22)}=0.07, p=0.94$; for missed trials: $t_{(22)}=0.87$, $p=0.40)$. The reaction times for correct responses were slightly but significantly longer for visual $(0.50 \mathrm{~s})$ than for auditory $(0.48$ s) trials (paired $t$ tests: $t_{(22)}=-2.26, p=0.03$ ).

\section{Acoustic data: otoacoustic emissions}

DPOAE measurements were optimized by individually adjusting the $\mathrm{f} 2$ frequencies. The resulting $\mathrm{f} 2$ frequencies were in the range of $1000-1888 \mathrm{~Hz}$ (mean $1568 \mathrm{~Hz}$ ). Figure $1 C$ gives an example of the spectrum of the acoustic recording in the ear canal, with the amplitude-modulated $\mathrm{f} 1$ tone, the pure $\mathrm{f} 2$ tone, and the resulting distortion product at frequency $2 \mathrm{f} 1-\mathrm{f} 2$ visible as distinct peaks.

Figure $2 A$ shows the DPOAE time course for the Attend Visual and Attend Auditory conditions averaged across all subjects. Directly after stimulus onset, DPOAE level showed a clear level decrease (onset adaptation). In the Attend Auditory condition, 


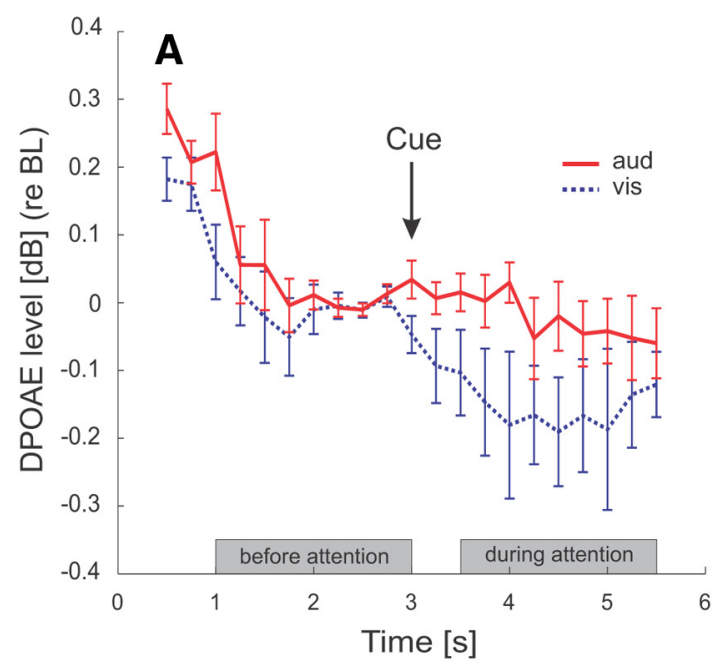

B

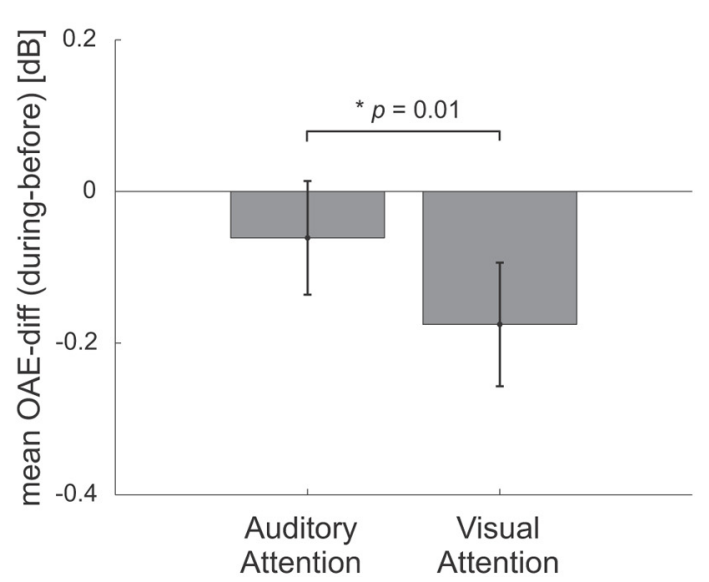

Figure 2. Impact of intermodal attention on DPOAEs. A, Averaged time course of DPOAE level across the task duration ( $n=23$, mean \pm SE). Red solid line, auditory task; blue dotted line, visual task. The DPOAElevel is depicted relative to baseline. $B$, Mean DPOAElevel change during attention compared with the time window before attention $(n=23, \pm S E$. The time windows are indicated by gray boxes in $\boldsymbol{A}$. There was a significant difference between the auditory and visual conditions ( $t$ test, $p=0.01$ ).

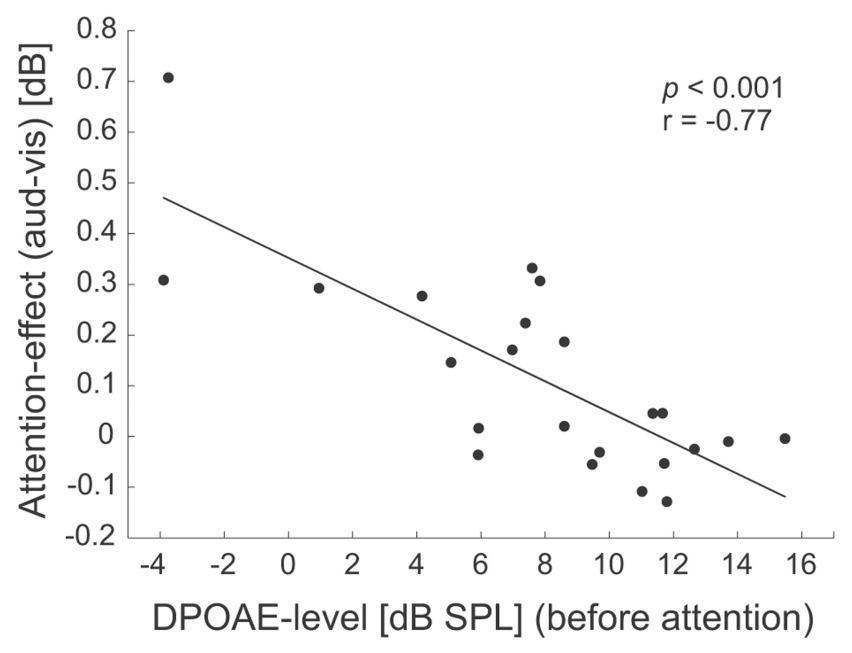

Figure 3. Correlation between attention effect and individual DPOAE base level. The individual difference of the DPOAE level change in auditory versus visual trials and the subjects' preattentional DPOAE level was negatively correlated $(r=-0.77 ; p<0.001)$.

DPOAE levels remained at baseline level after the presentation of the cue, whereas during visual attention, a distinct DPOAE level decrease was observed in the period of focused attention. Quantifying this effect of focused intermodal attention by calculating the DPOAE change during selective attention compared with precue periods (Fig. $2 B$ ) revealed a significant level decrease of $\sim 0.2 \mathrm{~dB}$ for the visual condition (one-sample $t$ test vs zero, $t_{(22)}=$ $-2.1511, p=0.0427)$, but nearly no change for the auditory condition (n.s. vs zero, $t_{(22)}=-0.8149 p=0.4239$ ). The level change in the period of focused attention compared with the period before focused attention differed significantly between the conditions Attend Auditory and Attend Visual (paired $t$ test: $\left.t_{(22)}=2.80, p=0.0103\right)$.

Further analysis of the individual data revealed a strong negative correlation between the general DPOAE level, measured as the mean DPOAE level before attention, and the attention effect, defined as the difference between the level change during auditory and during visual attention. As shown in Figure 3, a lower precue DPOAE level was associated with a more pronounced attention effect ( $r=-0.77, p<0.001$, Student's $t$ test).
Electrophysiological data: auditory steady-state responses Maximal $40 \mathrm{~Hz}$ ASSR power was observed at frontocentral sensors. To compare the $40 \mathrm{~Hz}$ ASSR responses between the conditions of visual and auditory attention, the time period before the informative cue, the time from 1 to $3 \mathrm{~s}$ after stimulus onset, served as a baseline. Figure 4 illustrates the results of auditory steadystate responses, averaged across all subjects. The topographic scalp distribution of the ASSR (Fig. $4 A$ ) showed a higher ASSR power at frontocentral sensors for the auditory than for the visual attention condition. Statistical comparison of the ASSR power between the visual and auditory conditions in the period of selective attention (0.5-2.5 s after cue) revealed one cluster of electrodes with significant differences between conditions $(p=0.03)$ at 4 time points (4.4-5 s after stimulus onset; Fig. $4 B$ ). Investigation of the time course of the ASSR power of this cluster of electrodes during the task revealed an increase of ASSR during auditory attention in the time after the informative cue indicating the to-be-attended modality (Fig. 4C).

\section{Electrophysiological data: alpha activity}

The predominant frequency band of the induced oscillatory activity was in the alpha range $(8-14 \mathrm{~Hz})$. We compared alpha activity in a time period requiring focused attention to either the visual or the auditory modality (0.5-2.5 s after cue) by cluster-based permutation tests and found two clusters of electrodes where alpha activity was significantly lower $(p<$ 0.01 ) for the visual than for the auditory trials (Fig. 5A). The first cluster was located over the occipitoparietal cortex and the second over the left frontal cortex. The difference in alpha activity between trials requiring auditory and visual attention began shortly after the presentation of the informative cue (Fig. $5 B$ ). Task onset and the presentation of the informative cue led to a distinct decrease in alpha power in both conditions. After cue presentation, alpha power remained at a low level for the rest of the task duration in the visual condition, whereas in the auditory condition, the alpha power increased after $\sim 1$ s to values above the precue level.

\section{Discussion}

The present result of different distortion product otoacoustic emission levels during visual attention compared with auditory 
A

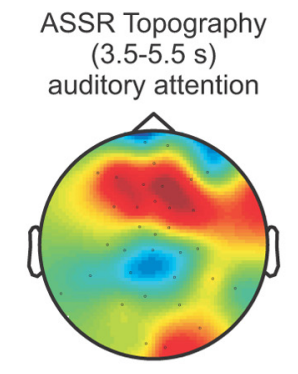

B Sensor Statistic $(p=0.03)$

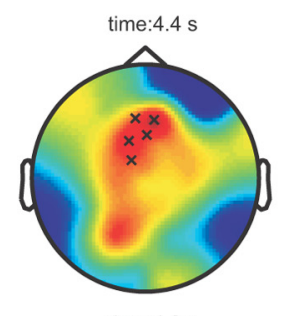

time: $4.8 \mathrm{~s}$

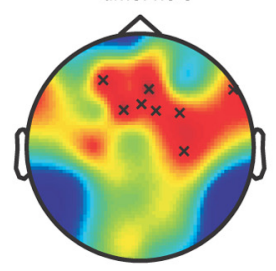

ASSR Topography

auditory attention

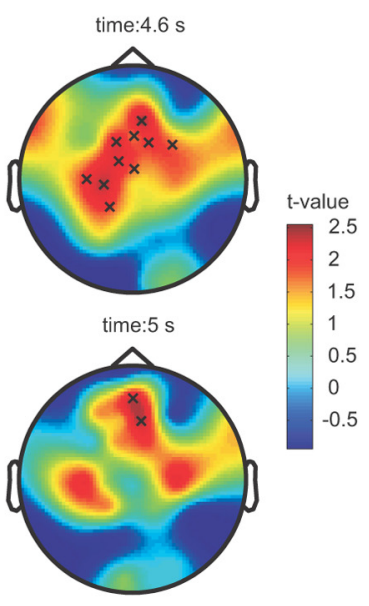

ASSR Topography

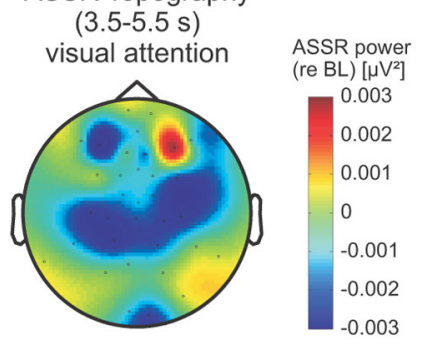

C

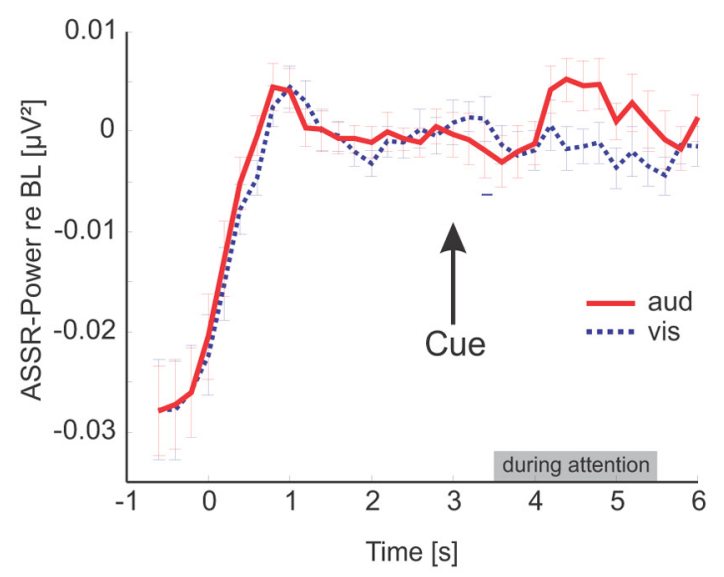

Figure 4. Auditory steady-state responses during auditory and visual attention. $A$, Topographical distribution of the $40 \mathrm{~Hz}$ ASSR during the period of focused attention averaged across all subjects for auditory (left) and visual (right) trials relative to the baseline period before the cue. B, Statistical contrast between conditions ( $t$ values). Electrodes of the significant cluster ( $p=0.03$ ) are marked with crosses. C, Time course of the ASSR power at cluster electrodes, mean \pm SE. Red solid line, auditory trials; blue dotted line, visual trials.

A

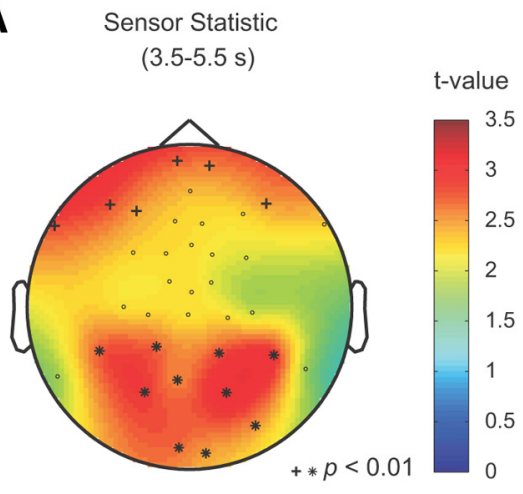

B

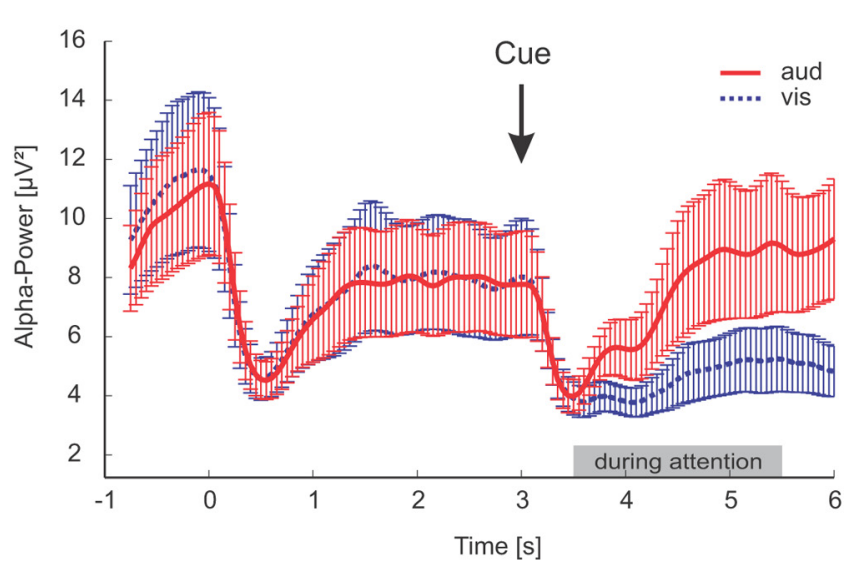

Figure 5. Attentional modulation of alpha activity $(8-14 \mathrm{~Hz}) . A$, Topographic representation of statistical differences ( $t$ values) between alpha activity during auditory and visual attention in the period $0.5-2.5 \mathrm{~s}$ after cue presentation. Electrodes of the two significant clusters are marked (first cluster at occipitoparietal sites: ${ }^{*} p<0.01$, second cluster at left frontal electrodes; $+p<0.01$ ). $B$, Time course of alpha power during the task. Averaged values are depicted for the occipitoparietal cluster electrodes for all subjects ( $n=23$, mean \pm SE). Red solid line, auditory trials; blue dotted line, visual trials.

attention demonstrates an influence of intermodal attention on the cochlea as the most peripheral stage of auditory processing. Given the known anatomical structures, the neural substrate of this effect must be the MOC efferent pathway, which is part of the corticofugal system and can modulate the cochlear mechanical state by innervating the outer hair cells directly.
We found a reduction of the otoacoustic emission level during visual attention, which might reflect a reduction of sound amplification in the cochlea. This would be consistent with an inhibition of the irrelevant and distracting acoustic stimulus during visual selective attention. Several previous studies have also found reduced otoacoustic emission amplitudes when human 
subjects were engaged in a visual task (Puel et al., 1988; Meric and Collet, 1992; Froehlich et al., 1993). However, in these studies, OAEs were compared between longer periods of visual attention and passive listening, so the observed differences might have been attributable to nonspecific arousal differences. In the present paradigm, we ensured comparable arousal states in both conditions using a trial-wise cueing paradigm. This design also controlled for slow drifts of the DPOAE level over the course of the experiment because we compared the level before and after the informative cue for each trial separately. Moreover, to ensure comparable difficulties between conditions and across subjects, we used an adaptive procedure producing equal hit rates.

Two recent studies using DPOAE found the opposite pattern compared with ours: a reduction of DPOAE level during auditory attention (Smith et al., 2012; Srinivasan et al., 2012). When discussing these contradictory results, it is important to consider the indirect nature of otoacoustic emission measurements. DPOAEs are generated as an acoustic byproduct of the nonlinear amplification in the outer hair cells and show complex and nonlinear characteristics. Small changes in the amplification process mediated by the MOC efferents might elicit level deviations in both directions depending on the choice of DPOAE stimulus frequencies and levels in the different studies. Significant changes of the DPOAE level in both directions during the activation of the olivocochlear efferents due to contralateral acoustic stimulation are described for humans and animals (Müller et al., 2005; Abel et al., 2009; Althen et al., 2012) and could be explained by models assuming shifts of the operating point of the cochlear amplifier (Abel et al., 2009). Delano et al. (2007) investigated the influence of selective attention in behaving chinchillas in a cueing paradigm and found, similarly to our results, a reduction of cochlear sensitivity (reduction of compound action potential amplitude) during visual attention after an informative cue. Also equivalent to our results, this study found no change of the cochlear response during auditory attention. Investigating attention effects in a cueing paradigm allows a very well controlled comparison between periods before and during attention; slow changes of cochlear response level and fluctuations of arousal that might occur during long-lasting block designs can be ruled out. Cueing is widely used in behavioral and neuroimaging studies in human, but our study was the first to apply cueing for the analysis of peripheral effects of selective attention in humans.

Interestingly, we found a negative correlation between the individual DPOAE base level and the magnitude of the attention effect. Subjects with lower DPOAE levels showed the clearest DPOAE modulation during selective attention. Two considerations might explain this observation. First, the olivocochlear efferent modulation of DPOAE has been shown to be more pronounced for low-level stimulation, when the cochlear amplifier is particularly important (Robles and Ruggero, 2001; Ren et al., 2011). Therefore, low DPOAE levels could correspond to the optimal operating range of the cochlear amplifier and thus high sensitivity for efferent modulation. Conversely, the stimulation level was constant for all subjects and a low DPOAE level might rather indicate low hearing sensitivity (Boege and Janssen, 2002). Subjects with higher hearing thresholds and lower DPOAE levels could possibly benefit more from an early attentional impact on cochlear amplification and would therefore use such a modulation more markedly.

In our study, like in previous ones, the observed OAE level change during focused attention appeared rather small. However, considering the nonlinear course of DPOAE input-output functions, small alterations might correspond to bigger differ- ences in the evoking stimulus level, so small changes in the DPOAE level as an indirect indicator of cochlear amplification might resemble bigger, highly relevant changes in auditory processing. Independently of effect direction and the rather small effect size, it is important that the present study, as well as previous ones, demonstrated a significant influence of task demand on the mechanics of the cochlea.

The novel approach of our study was the combination of DPOAE and EEG measurements to assess attentional effects simultaneously on different stages of the auditory system. By measuring auditory steady-state responses, we monitored the effects of attention at early cortical stages. The $40 \mathrm{~Hz}$ ASSR showed a frontocentral distribution, similar to the ASSR distribution described by others (Griskova et al., 2007; Saupe et al., 2009; de Jong et al., 2010). Its topography matched the assumed source of ASSRs in the primary auditory cortex located in Heschl's gyrus. As expected, the ASSR varied, with task demands showing significantly higher power during auditory than during visual attention. This is consistent with former studies, such as one by Saupe et al. (2009, 2009b), who found a significant enhancement of ASSR during auditory attention compared with visual attention in an intermodal selective attention task. Similarly, Gander et al. (2010) described changes in ASSR power dependent on the attentional demand (auditory vs visual attention), but only when attention was required for brief $(1 \mathrm{~s})$ but not for long $(2 \mathrm{~min})$ time intervals. In an earlier study, no effects of attention on the ASSR were seen (Linden et al., 1987). Apparently, an attentional modulation of ASSR at primary auditory cortex is influenced by the specific experimental design and the difficulty and exact time course of the task. It has been suggested that differences in ASSR levels might be obvious only when the target event is defined by a change of the modulation frequency itself (Ross et al., 2004; Skosnik et al., 2007; Saupe et al., 2009b), which was not the case in our paradigm and could explain why the present effect, even though it reached statistical significance, was rather small.

The analysis of induced oscillatory activity in the EEG provided a further control for the attentional demands of the task. We found a suppression of alpha-band activity in the visual trials compared with the auditory trials in the period of selective attention for a cluster of occipital electrodes. Recent studies have suggested an active role of cortical alpha oscillations for cognitive functions, including short-term memory (Kaiser et al., 2007) and attention (Foxe and Snyder, 2011; Händel et al., 2011; Mazaheri et al., 2014). In the context of selective attention, alpha-band activity is suggested to reflect a suppression mechanism. Our results are consistent with this view: during selective attention to visual stimuli, alpha power decreased in occipital regions that most likely correspond to early visual cortex. Thus neural suppression by alpha oscillations is reduced, whereas during auditory attention, alpha power as an attentional suppressor is increased in this area, corresponding to a suppression of the distracting visual input during focusing on auditory stimuli. Changes in alpha-band power have been shown for intermodal attention (Foxe et al., 1998) and for intrasensory spatial and feature-based attention in different sensory domains (Fu et al., 2001; Thut et al., 2006; Klimesch et al., 1998) and seem to reflect a general mechanism for attentional gating when competing sensory input needs to be ignored or selected. The typical alpha effect in the present study further confirmed the effectiveness of our experimental paradigm for the investigation of intermodal attention. The effects at the level of the cochlea are therefore unlikely to reflect arousal differences. 
The interindividual variability was rather high in all measures (DPOAE, ASSR, alpha power) and we failed to find correlations between them. This may be attributable to the fact that the different effects we show correspond to different processes associated to selective attention (cochlea: suppression of the distractor in the auditory system; primary auditory cortex: enhancement of the attended stimulus; alpha: suppression of distractor processing in the visual system) and thus do not depend on each other directly. In conclusion, we observed a significant influence of intermodal selective attention at different stages of the auditory processing hierarchy. Attentional modulation of auditory processing was found already at the level of the cochlea. This supports the hypothesis of a cognitive impact on the micromechanical mechanisms in the inner ear, probably mediated by the olivocochlear efferent system. However, further studies will be necessary to integrate the present findings in the general function of the olivocochlear efferent system for human hearing.

\section{References}

Abel C, Wittekindt A, Kössl M (2009) Contralateral acoustic stimulation modulates low-frequency biasing of DPOAE: efferent influence on cochlear amplifier operating state? J Neurophysiol 101:2362-2371. Medline

Althen H, Wittekindt A, Gaese B, Kössl M, Abel C (2012) Effect of contralateral pure tone stimulation on distortion emissions suggests a frequencyspecific functioning of the efferent cochlear control. J Neurophysiol 107: 1962-1969. CrossRef Medline

Ashmore J (2008) Cochlear outer hair cell motility. Physiol Rev 88:173-210. CrossRef Medline

Avan P, Bonfils P (1992) Analysis of possible interactions of an attentional task with cochlear micromechanics. Hear Res 57:269-275. CrossRef Medline

Boege P, Janssen T (2002) Pure-tone threshold estimation from extrapolated distortion product otoacoustic emission I/O-functions in normal and cochlear hearing loss ears. J Acoust Soc Am 111:1810-1818. CrossRef Medline

Carrasco M, Ling S, Read S (2004) Attention alters appearance. Nat Neurosci 7:308-313. CrossRef Medline

Connolly JF, Aubry K, McGillivary N, Scott DW (1989) Human brainstem auditory evoked potentials fail to provide evidence of efferent modulation of auditory input during attentional tasks. Psychophysiology 26:292-303. CrossRef Medline

Couperus JW, Mangun GR (2010) Signal enhancement and suppression during visual-spatial selective attention. Brain Res 1359:155-177. CrossRef Medline

de Boer J, Thornton AR (2007) Effect of subject task on contralateral suppression of click evoked otoacoustic emissions. Hear Res 233:117-123. CrossRef Medline

de Jong R, Toffanin P, Harbers M (2010) Dynamic crossmodal links revealed by steady-state responses in auditory-visual divided attention. Int J Psychophysiol 75:3-15. CrossRef Medline

Delano PH, Elgueda D, Hamame CM, Robles L (2007) Selective attention to visual stimuli reduces cochlear sensitivity in chinchillas. J Neurosci 27: 4146-4153. CrossRef Medline

Engelien A, Schulz M, Ross B, Arolt V, Pantev C (2000) A combined functional in vivo measure for primary and secondary auditory cortices. Hear Res 148:153-160. CrossRef Medline

Foxe JJ, Snyder AC (2011) The role of alpha-band brain oscillations as a sensory suppression mechanism during selective attention. Front Psychol 2:154. CrossRef Medline

Foxe JJ, Simpson GV, Ahlfors SP (1998) Parieto-occipital $\sim 10 \mathrm{~Hz}$ activity reflects anticipatory state of visual attention mechanisms. Neuroreport 9:3929-3933. CrossRef Medline

Fritz JB, Elhilali M, David SV, Shamma SA (2007) Auditory attentionfocusing the searchlight on sound. Curr Opin Neurobiol 17:437-455. CrossRef Medline

Froehlich P, Collet L, Morgon A (1993) Transiently evoked otoacoustic emission amplitudes change with changes of directed attention. Physiol Behav 53:679-682. CrossRef Medline

Fu KM, Foxe JJ, Murray MM, Higgins BA, Javitt DC, Schroeder CE (2001) Attention-dependent suppression of distracter visual input can be cross- modally cued as indexed by anticipatory parieto-occipital alpha-band oscillations. Brain Res Cogn Brain Res 12:145-152. CrossRef Medline

Gander PE, Bosnyak DJ, Roberts LE (2010) Evidence for modality-specific but not frequency-specific modulation of human primary auditory cortex by attention. Hear Res 268:213-226. CrossRef Medline

Giard MH, Fort A, Mouchetant-Rostaing Y, Pernier J (2000) Neurophysiological mechanisms of auditory selective attention in humans. Front Biosci 5:D84-D94. Medline

Green TJ, McKeown JD (2001) Capture of attention in selective frequency listening. J Exp Psychol Hum Percept Perform 27:1197-1210. CrossRef Medline

Griskova I, Morup M, Parnas J, Ruksenas O, Arnfred SM (2007) The amplitude and phase precision of $40 \mathrm{~Hz}$ auditory steady-state response depend on the level of arousal. Exp Brain Res 183:133-138. CrossRef Medline

Griskova I, Morup M, Parnas J, Ruksenas O, Arnfred SM (2009) Two discrete components of the $20 \mathrm{~Hz}$ steady-state response are distinguished through the modulation of activation level. Clin Neurophysiol 120:904909. CrossRef Medline

Guinan JJ (1996) Physiology of olivocochlear efferents. In: The cochlea (Dallos P, Popper AN, Fay RR, eds), pp 435-502. New York: Springer.

Gutschalk A, Mase R, Roth R, Ille N, Rupp A, Hähnel S, Picton TW, Scherg M (1999) Deconvolution of $40 \mathrm{~Hz}$ steady-state fields reveals two overlapping source activities of the human auditory cortex. Clin Neurophysiol 110:856-868. CrossRef Medline

Händel BF, Haarmeier T, Jensen O (2011) Alpha oscillations correlate with the successful inhibition of unattended stimuli. J Cogn Neurosci 23: 2494-2502. CrossRef Medline

Kaiser J, Heidegger T, Wibral M, Altmann CF, Lutzenberger W (2007) Alpha synchronization during auditory spatial short-term memory. Neuroreport 18:1129-1132. CrossRef Medline

Karns CM, Knight RT (2009) Intermodal auditory, visual, and tactile attention modulates early stages of neural processing. J Cogn Neurosci 21:669683. CrossRef Medline

Kemp DT (2007) Otoacoustic emissions: concepts and origins. In: Active processes and otoacoustic emissions in hearing (Manley GA, Fay RR, Popper AN, eds), pp 1-38. New York: Springer.

Klimesch W (2012) alpha-band oscillations, attention, and controlled access to stored information. Trends Cogn Sci 16:606-617. CrossRef Medline

Klimesch W, Doppelmayr M, Russegger H, Pachinger T, Schwaiger J (1998) Induced alpha band power changes in the human EEG and attention. Neurosci Lett 244:73-76. CrossRef Medline

Linden RD, Picton TW, Hamel G, Campbell KB (1987) Human auditory steady-state evoked potentials during selective attention. Electroencephalogr Clin Neurophysiol 66:145-159. CrossRef Medline

Lukas JH (1980) Human auditory attention: the olivocochlear bundle may function as a peripheral filter. Psychophysiology 17:444-452. CrossRef Medline

Maison S, Micheyl C, Collet L (2001) Influence of focused auditory attention on cochlear activity in humans. Psychophysiology 38:35-40. CrossRef Medline

Maris E, Oostenveld R (2007) Nonparametric statistical testing of EEG- and MEG-data. J Neurosci Methods 164:177-190. CrossRef Medline

Mazaheri A, van Schouwenburg MR, Dimitrijevic A, Denys D, Cools R, Jensen O (2014) Region specific modulations in oscillatory alpha activity serves to facilitate processing in the visual and auditory modalities. Neuroimage 87:356-362. CrossRef Medline

Meric C, Collet L (1992) Visual attention and evoked otoacoustic emissions: A slight but real effect. Int J Psychophysiol 12:233-235. CrossRef Medline

Meric C, Collet L (1994) Attention and otoacoustic emissions: a review. Neurosci Biobehav Rev 18:215-222. CrossRef Medline

Michie PT, LePage EL, Solowij N, Haller M, Terry L (1996) Evoked otoacoustic emissions and auditory selective attention. Hear Res 98:54-67. CrossRef Medline

Müller J, Janssen T, Heppelmann G, Wagner W (2005) Evidence for a bipolar change in distortion product otoacoustic emissions during contralateral acoustic stimulation in humans. J Acoust Soc Am 118:3747-3756. CrossRef Medline

Oostenveld R, Fries P, Maris E, Schoffelen JM (2011) FieldTrip: open source software for advanced analysis of MEG, EEG, and invasive electrophysiological data. Comput Intell Neurosci 2011:156869. CrossRef Medline

Picton TW, Hillyard SA, Galambos R, Schiff M (1971) Human auditory 
attention: a central or peripheral process? Science 173:351-353. CrossRef Medline

Posner MI (1980) Orienting of attention. Quarterly Journal of Experimental Psychology 32:3-25. CrossRef Medline

Puel JL, Bonfils P, Pujol R (1988) Selective attention modifies the active micromechanical properties of the cochlea. Brain Res 447:380-383. CrossRef Medline

Ren T, He W, Porsov E (2011) Localization of the cochlear amplifier in living sensitive ears. PLoS One 6:e20149. CrossRef Medline

Robles L, Ruggero MA (2001) Mechanics of the mammalian cochlea. Physiol Rev 81:1305-1352. Medline

Ross B, Picton TW, Herdman AT, Pantev C (2004) The effect of attention on the auditory steady-state response. Neurol Clin Neurophysiol 2004:22. Medline

Russell IJ, Lukashkin AN (2007) Cellular and molecular mechanisms in the efferent control of cochlear nonlinearities. In: Active processes and otoacoustic emissions in hearing (Manley GA, Fay RR, Popper AN, eds), pp 343-379. New York: Springer.

Saupe K, Schröger E, Andersen SK, Müller MM (2009) Neural mechanisms of intermodal sustained selective attention with concurrently presented auditory and visual stimuli. Front Hum Neurosci 3:58. CrossRef Medline
Saupe K, Widmann A, Bendixen A, Müller MM, Schröger E (2009b) Effects of intermodal attention on the auditory steady-state response and the event-related potential. Psychophysiology 46:321-327. CrossRef Medline

Skosnik PD, Krishnan GP, O’Donnell BF (2007) The effect of selective attention on the gamma-band auditory steady-state response. Neurosci Lett 420:223-228. CrossRef Medline

Smith DW, Aouad RK, Keil A (2012) Cognitive task demands modulate the sensitivity of the human cochlea. Front Psychol 3:30. CrossRef Medline

Srinivasan S, Keil A, Stratis K, Woodruff Carr KL, Smith DW (2012) Effects of cross-modal selective attention on the sensory periphery: Cochlear sensitivity is altered by selective attention. Neuroscience 223:325-332. CrossRef Medline

Thut G, Nietzel A, Brandt SA, Pascual-Leone A (2006) Alpha-band electroencephalographic activity over occipital cortex indexes visuospatial attention bias and predicts visual target detection. J Neurosci 26:9494-9502. CrossRef Medline

Wagner W, Heppelmann G, Vonthein R, Zenner HP (2008) Test-retest repeatability of distortion product otoacoustic emissions. Ear Hear 29:378 391. CrossRef Medline

Ward LM (1997) Involuntary listening aids hearing. Psychological Science 8:112-118. CrossRef 